\title{
La métastase cancéreuse
}

Les mécanismes cellulaires par lesquels une cellule tumorale peut acquérir un potentiel métastatique sont sans aucun doute variés : l'activation ou la répression transitoire de gènes, due peut-être à l'instabilité génétique inhérente aux cellules tumorales, peut conduire à la synthèse de protéases, à la sécrétion de facteurs de mobilité et à la perte d'adhérence intercellulaire. La détermination des mécanismes de production et de développement des métastases aurait sans nul doute d'importantes implications diagnostiques et thérapeutiques.

Brigitte Boyer

Jacqueline Jouanneau Gordon Tucker Ana Maria Vallés Xavier Sastre Ginette Moens Jean-Paul Thiéry

\section{ADRESSES}

B. Boyer : docteur en médecine, docteur ès sciences. J. Jouanneau : docteur ès sciences. G. Tucker : ingénieur. A.M. Vallés : docteur ès sciences. G. Moens : technicienne. J.-P. Thiéry : docteur ès sciences, directeur du laboratoire de physiopathologie du développement. Laboratoire de physiopathologie du développement, Cnrs URA 1337 et École normale supérieure, 46, rue d'Ulm, 75006 Paris, France. $\mathrm{X}$. Sastre : docteur en médecine, médecin hospitalier. Laboratoire d'histopathologie, Institut Curie, 26, rue d'Ulm, 75230 Paris Cedex 05, France. a métastase (du grec metastasis: déplacement) qui correspond à un changement dans le siège d'une maladie, appartient essentiellement au domaine de la cancérologie où elle représente l'évolution, souvent dramatique, de nombreuses tumeurs malignes. Un exemple de dissémination métastatique à partir d'un cancer mammaire est schématisé sur la figure 1, page suivante. La difficulté à soigner les métastases cancéreuses provient de causes différentes. D'une part, ce sont des lésions multifocales qui ne peuvent donc pas être réséquées chirurgicalement, comme le sont, souvent avec un certain succès, les cancers primaires. Les seules armes thérapeutiques dont on dispose alors sont la radiothérapie et la chimiothérapie, qui échouent souvent du fait de l'émergence de cellules cancéreuses résistantes à ces traitements et, plus particulièrement, à la chimiothérapie anticancéreuse. D'autre part, il n'existe pas, à l'heure actuelle, de détection précoce ni de traitement préventif des métastases que l'on pourrait appliquer dans les cas de cancer primaire déclaré. Enfin, des métastases cancéreuses peuvent se développer avant même que le diagnostic de cancer primaire ait pu être porté. Il semble bien, en effet, que le processus métastatique puisse prendre place très précocement au cours du développement d'un cancer primaire: une tumeur de moins d'un gramme, cliniquement silencieuse, est indétectable, même par l'imagerie médicale la plus sophistiquée, mais cette tumeur qui contient quelques millions de cellules a déjà pu produire des cellules métastatiques, qui auront formé des micrométastases, indétectables elles aussi.

La compréhension du phénomène métastatique représente un objectif capital dans la recherche cancérologique, puisqu'elle pourrait à terme déboucher sur des méthodes diagnostiques et thérapeutiques mieux adaptées au cas particulier de la cellule cancéreuse métastatique [1]. 


\section{Métastases des cancers du sein}

\section{RÉFÉRENCES}

1. Salomon JC. Les métastases des cancers La Recherche 1982 ; 129 : 52-60.

2. Tarin D, Price JE, Kettlewell MGW, Souter RG, Vass ACR, Crossley B. Mechanisms of human tumor metastasis studied in patients with peritoneovenous shunts. Cancer Res 1984 ; 44 : 3584-92.

3. Paget S. The distributions of secondary growths in cancer of the breast. Lance $1889 ; 1: 571-3$

4. Weiss L. Random and nonrandom processes in metastasis, and metastatic efficiency. Invasion Metastasis 1983 ; 3 : 193-208.

5. Fidler IJ, Kripke ML. Metastasis results from preexistencing variant cells within a malignant tumor. Science 1977 ; 197 : 893-5.

6. Fidler IJ, Hart IR. Biological diversity in metastasis and metastatic neoplasms : origins and implications. Science 1982 ; 217 : 998-1003.

7. Marx J. Many gene changes found in cancer. Cancer 1989; 246 : 1386-8.

8. Kerbel RS. Towards an understanding of the molecular basis of the metastatic phenotype. Invasion Metastasis $1989 ; 9$ : 329-37.

9. Bishop JM. Cellular oncogenes and retrovirus. Ann Rev Biochem 1983; 52 : 301-54

10. Barbacid M. Ras genes. Ann Rev Biochem 1987 ; 56 : 779-827.

11. Mareel MM, Van Roy FM. Are oncogenes involved in invasion and metastasis. Anti-Cancer Res 1986 ; 6 : 419-36.

12. Sager R. Tumor suppressor genes : the puzzle and the promise. Science $1989 ; 246$ : 1406-12.

13. Bevilacqua $G$, Sobel ME, Liotta LA Steeg PS. Association of low nm23 RNA levels in human primary infiltrating ductal breast carcinomas with lymph node involvement and other histopathological indicators of high metastatic potential. Cancer Res $1989 ; 49$ : 5185-90.

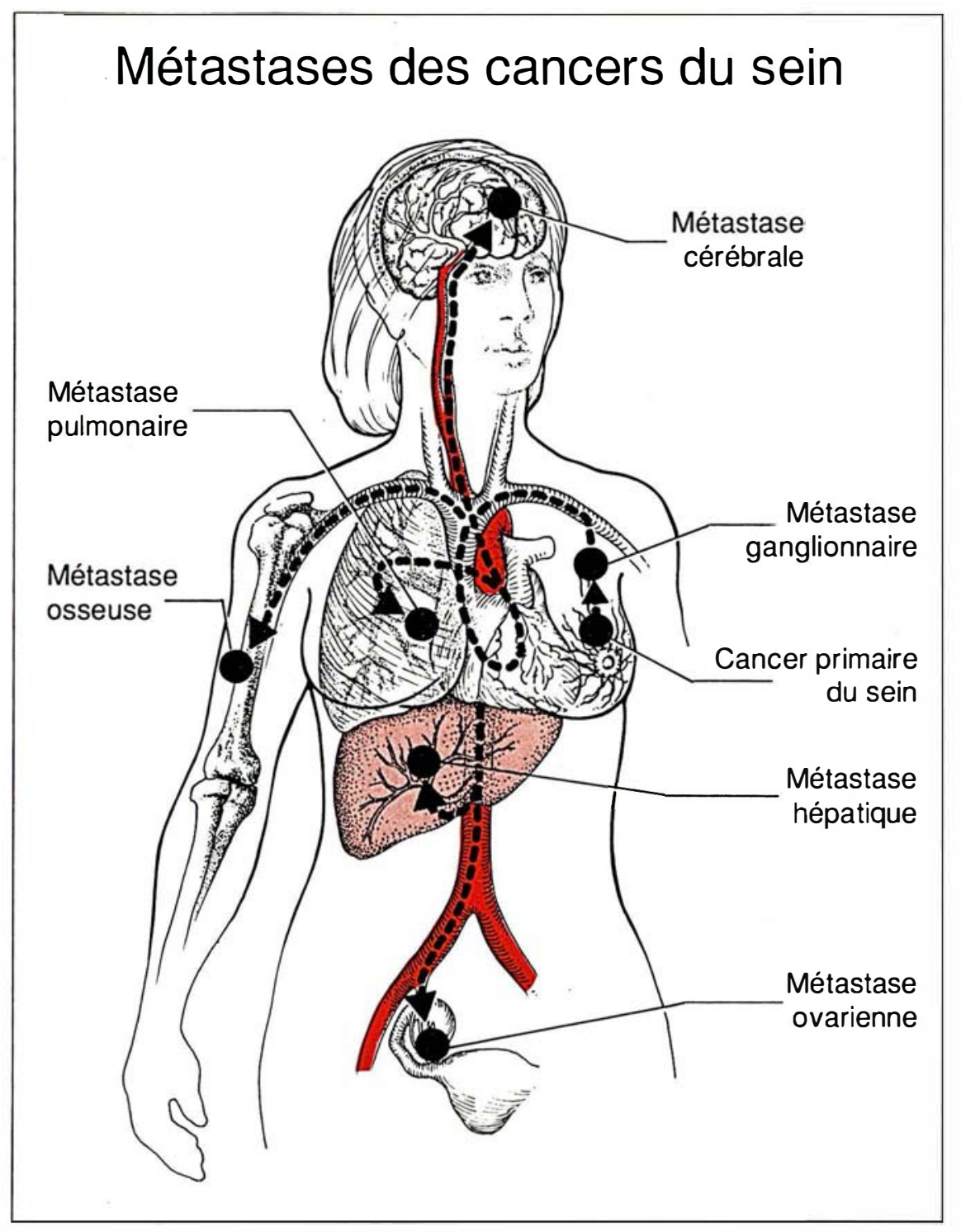

Figure 1. Voies de dissémination et sites d'implantation des métastases du cancer mammaire. ISchéma reproduit avec I'autorisation du Dr. G.L. Nicolson).

L'étude du processus métastatique s'est développée selon deux axes principaux : mise au point de modèles animaux expérimentaux et analyse anatomopathologique de tumeurs humaines et animales.

\section{Modèles expérimentaux}

La métastase expérimentale, qui consiste à injecter des cellules tumorales directement dans la circulation sanguine et, après sacrifice de l'animal, à estimer le nombre de métastases pulmonaires formées, a été très utilisée dans la recherche fondamentale. Le bien-fondé de cette méthode, apparemment artificielle, repose sur des observations cliniques indéniables: pour des raisons thérapeutiques, des dérivations du liquide ascitique vers la circulation veineuse ont parfois été réalisées chez des patients atteints de tumeur ascitique, conduisant à l'introduction massive de cellules tumorales dans le sang. On a 
pu alors constater que, pendant le temps de survie, parfois important, de ces patients, aucune métastase ne se formait [2]. C'est dire que l'introduction massive de cellules cancéreuses dans la circulation ne conduit pas nécessairement à la formation de métastases. Cette méthode, dite de métastase expérimentale, peut être donc utilisée pour tester certaines propriétés des cellules tumorales s'exprimant lors des dernières étapes du processus métastatique. Malgré tout, afin de reproduire plus fidèlement la métastase naturelle, des méthodes de métastase spontanée ont été mises au point : les cellules cancéreuses sont alors injectées en souscutané chez l'animal et la capacité à métastaser est évaluée, souvent après ablation de la tumeur primaire, par le nombre de métastases (en général pulmonaires) qui se sont développées. Enfin, la tendance expérimentale actuelle est de tenir compte au maximum de la spécificité tissulaire de la tumeur et d'in jecter les cellules cancéreuses dans l'organe dont elles sont dérivées, afin de reproduire d'aussi près que possible les conditions naturelles (Dr Fidler, communication personnelle).

\section{Analyses anatomopathologiques}

Les travaux menés depuis plus d'un siècle ont permis de définir au moins 11 étapes successives que les cellules tumorales doivent franchir pour s'échapper de la tumeur primaire et se fixer dans un organe situé à distance du foyer primaire (figures $2 \mathrm{ci}$ contre et 3, page suivante). Puisque les carcinomes, dérivés des tissus épithéliaux, forment $90 \%$ des tumeurs humaines, ils seront plus particulièrement étudiés ici. Dans le cas des épithéliomas, après une phase parfois prolongée de carcinome in situ, certaines cellules tumorales traversent la membrane basale après protéolyse de celle-ci ou arrêt de synthèse de ses composants. Les cellules cancéreuses pénètrent alors dans le stroma péritumoral et interagissent avec lui. La réaction stromale à la présence de cellules tumorales est caractérisée en particulier par un changement de composition de la matrice extracellulaire (figures $3 f$ et $3 g$ ) et l'apparition de $\mathrm{m} / \mathrm{s} n^{\circ} 5$ vol. 6 , mai 90

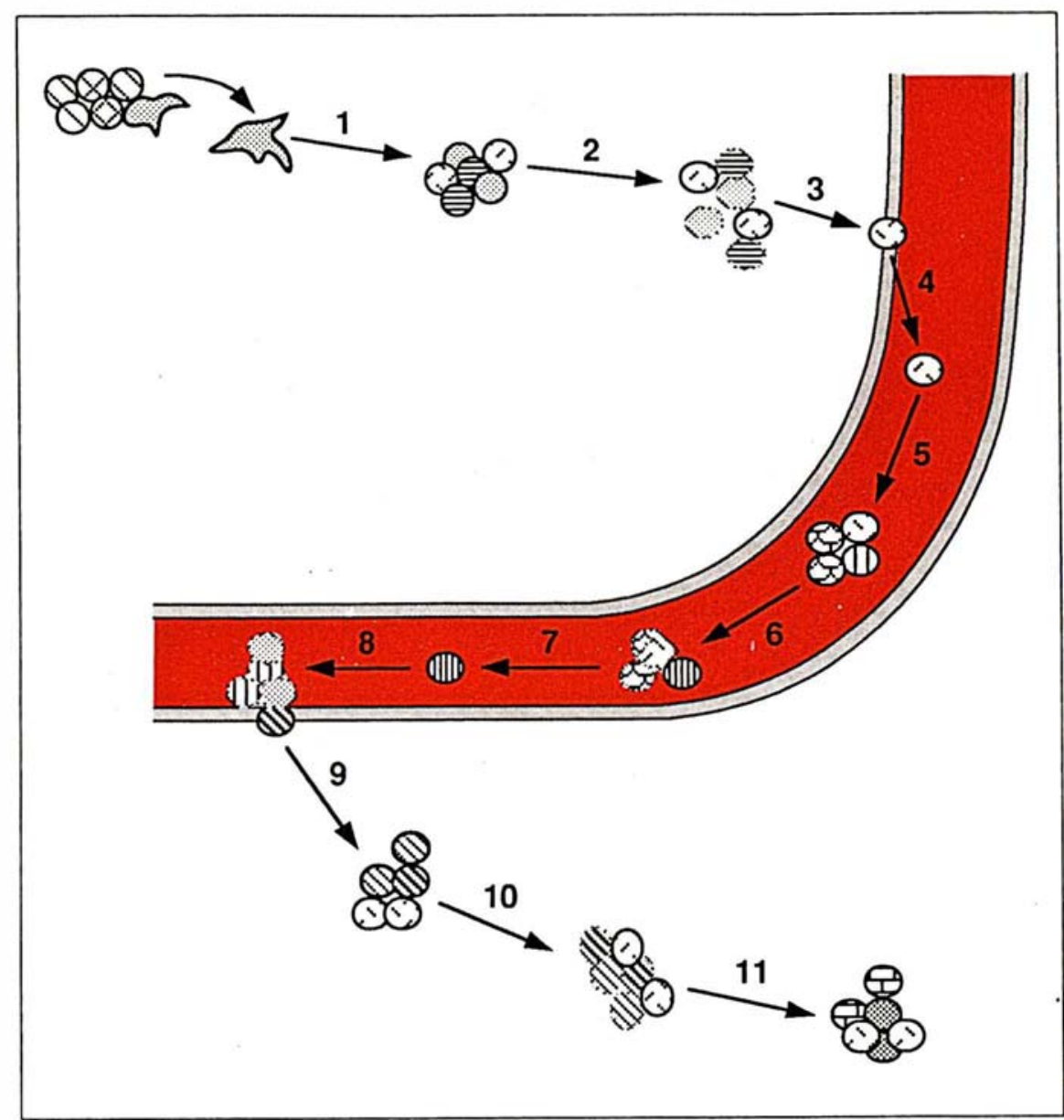

Figure 2. Un modèle possible du processus métastatique appliqué au cas des tumeurs solides. Une cellule lou un groupe de cellules) s'échappe de la tumeur primaire et prolifère (étape 1). Par le jeu des "mutations-sélections", un certain nombre de cellules meurent et seules subsistent celles qui s'adaptent aux conditions locales (étape 2). Ces cellules ont la capacité de franchir les parois des vaisseaux sanguins ou lymphatiques (étape 3), sont transportées dans les vaisseaux (étape 4) où elles peuvent éventuellement proliférer ou former des microagrégats (étape 5). Là encore, seules les cellules ayant un avantage sélectif (celles qui sont, en particulier, immunorésistantes) survivront (étape 6). Ces cellules s'arrêtent de migrer (étape 7l et se fixent ensuite à la paroi d'un vaisseau, formant une microembolie (étape 8). Les cellules ayant réussi à résister aux conditions locales et à franchir la paroi du capillaire, migrent dans le tissu interstitiel et $y$ prolifèrent (étape 9). Après un nouveau cycle de mutations-sélections (étape 10), une tumeur secondaire se reforme (étape 11).

fibroblastes d'un type particulier. Un certain nombre de cellules tumorales s'accumulent autour des vaisseaux et capillaires, dont certains ont été formés lors du processus de néoangiogenèse (c'est-à-dire de bourgeonnement de vaisseaux néoformés dérivant de vaisseaux péritumoraux), puis pénètrent dans ceux-ci et se trouvent, sous forme de cellules isolées ou de petits agrégats, transportées à distance par le flux sanguin ou lymphatique, ce qui permet une dissémination tumorale rapide. L'immobilisation des cellules cancéreuses se produit probablement dans les petits 


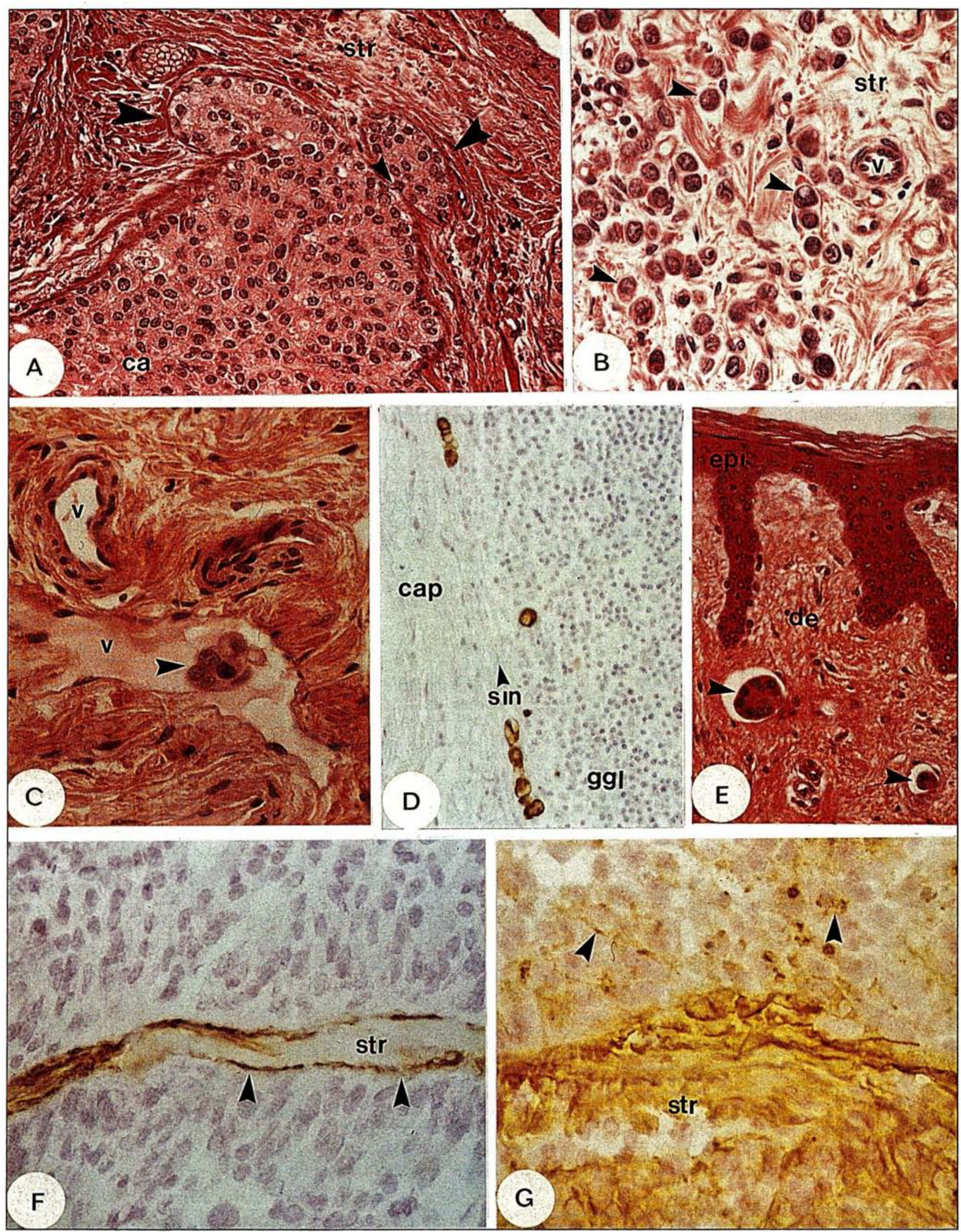


capillaires où le flux est faible. Elle est sans doute facilitée par la capacité des cellules tumorales à s'agréger pour former des microembolies, entourées d'un microcaillot de plaquettes et de fibrine. L'embolisation des cellules tumorales pourrait d'ailleurs permettre aux cellules situées à l'intérieur de l'agrégat d'échapper à la cytolyse provoquée par les lymphocytes $T$ ou les lymphocytes tueurs naturels. Après adhérence spécifique des cellules métastatiques aux cellules endothéliales entourant les capillaires du tissu cible, l'extravasation se produit, permettant l'envahissement stromal. Les cellules tumorales peuvent alors reformer une tumeur de structure comparable à celle de la tumeur primaire. Depuis 1889 , année où Paget, dans un article publié par le Lancet, lança sa célèbre hypothèse du seed and soil - ainsi nommée par analogie avec le fait qu'une graine (seed) ne peut pousser que dans un terrain (soil) qui lui est favorable -, il est clair que les foyers métastatiques ne siègent pas dans n'importe quel organe, et qu'on peut donc définir une certaine spécificité de l'organe cible qui est fonction du type de la cellule tumorale métastatique [3].

Chaque étape du processus métastatique représente un obstacle que seul un petit nombre de cellules, ayant pu s'adapter à l'environnement local, réussiront à franchir. Pour cette raison, Weiss qualifia le processus métastatique de phénomène hautement inefficace [4].

La probabilité qu'existent des cellules tumorales compétentes pour métastaser serait quasiment nulle si l'instabilité phénotypique des cellules tumorales ne permettait l'émergence, à tout moment et au hasard, de cellules ayant acquis de façon transitoire un pouvoir métastatique.

\section{Propriétés des cellules métastatiques}

- Instabilité génotypique et phénotypique. Cette propriété des cellules tumorales permet d'expliquer l'émergence, au sein d'une tumeur, de cellules à potentiel métastatique élevé qui seront sélectionnées positivement au cours du processus métastatique. L'expérimentation animale a ainsi permis de sélectionner des lignées tumorales au pouvoir métastatique nul, intermédiaire ou élevé. Ainsi, l'analyse d'un mélanome murin en culture (lignée B16), après plusieurs cycles d'injection dans la souris et de prélèvement des métastases pulmonaires formées, a permis de sélectionner un clone (B16-F1) à faible capacité métastatique et un clone (B16-F10) à pouvoir métastati- que élevé [5, 6]. Mais, mis en culture à long terme, le clone B16-F10 perd progressivement son potentiel métastatique et seules de nouvelles injections dans l'animal permettent alors d'enrichir la lignée en cellules très métastatiques. Cette approche expérimentale de la métastase a permis de démontrer clairement l'instabilité du phénotype métastatique des cellules tumorales. La progression tumorale allant de la prolifération bénigne à l'acquisition d'un pouvoir métastatique semble, par ailleurs, mettre en cause des mutations ponctuelles, des délétions ou des remaniements chromosomiques dont certains peuvent activer des protooncogènes [7].

- Oncogènes. Ces dernières années, la cancérologie fondamentale s'est enrichie de la découverte de gènes capables de contrôler l'expression du phénotype transformant. L'expression anormale de certains de ces gènes, appelés oncogènes, conduit à des cellules dont la croissance est partiellement indépendante des facteurs de croissance et qui peuvent alors entamer un processus de transformation néoplasique avec capacité de former une tumeur in vivo. Comme nous l'avons déjà mentionné, l'acquisition de propriétés métastatiques par des cellules tumorales s'est révélé être un processus complexe, dû à une cas-

$\triangleleft$ Figure 3. Analyse anatomo-pathologique des cancers mammaires. A. Épithélioma du sein micro-infiltrant : les cellules malignes prolifèrent à l'intérieur du canal galactophore (ca), dont elles obstruent la lumière, et commencent à envahir le tissu conjonctif péricanalaire. B. Épithélioma du sein de type lobulaire infiltrant : dans cette forme histologique particulière du cancer du sein, les cellules malignes apparaissent le plus souvent indépendantes les unes des autres et les contacts intercellulaires sont réduits ou absents. C. Embolie vasculaire des cellules malignes dans un cas de cancer du sein : les cellules malignes ont franchi la membrane basale des vaisseaux (v) et suivent le courant circulatoire. D. Phase initiale de l'infiltration métastatique d'un ganglion axillaire par des cellules malignes issues d'un épithélioma du sein de type lobulaire : les cellules néoplasiques colonisent les ganglions à partir des sinus sous-capsulaires externes (sin). Ces cellules ont été révélées grâce à la présence, dans leur cytoplasme, de cytokératines détectées à l'aide d'un anticorps spécifique marqué à la peroxydase. E. Infiltration de la peau par des cellules malignes issues d'un épithélioma du sein de type lobulaire : les cellules tumorales atteignent le derme (de) à partir des vaisseaux lymphatiques. F. Marquage de ED2 dans le cas d'un carcinome mammaire infiltrant. ED2 est une séquence de 90 acides aminés des fibronectines qui s'exprime surtout pendant le développement embryonnaire. La présence de variants de fibronectine contenant ED2 est difficile à détecter chez l'adulte, sauf dans le cas de la régénération tissulaire et dans les tumeurs [41, 42]. Ici, le marquage est limité au stroma (str), et principalement à la zone de contact avec les massifs néoplasiques. G. Marquage de la tenascine dans le cas de carcinome mammaire infiltrant : la tenascine est une glycoprotéine de la matrice extracellulaire qui joue un rôle modulateur de l'adhérence des cellules à la matrice. Elle est exprimée en très grandes quantités chez l'adulte au cours de la régénération et dans le stroma des tumeurs [43]. Ici, le marquage est présent dans le stroma, mais également dans certaines cellules épithéliales malignes, en particulier celles proches des travées fibreuses. (ca = canal galactophore; $v=$ vaisseau; cap = capsule ganglionnaire; $g g l=$ ganglion lymphatique $; \sin =$ sinus sous-capsulaire $;$ epi $=$ épiderme $;$ de $=$ derme.) 


\section{RÉFÉRENCES}

14. Rosengard AM, Krutzsch HC, Shearn A, et al. Reduced Nm23/Awd protein on tumour metastasis and aberrant Drosophila development. Nature 1989 ; 342 : 177-80.

15. Gherardi E, Gray J, Stoker M, Perryman M, Furlong R. Purification of scatter factor, a fibroblast-derived basic protein that modulates epithelial interactions and movement. Proc Nall Acad Sci USA 1989 ; 86 : 5844-8.

16. Grey AM, Schor AM, Rushton G, Ellis I, Schor SL. Purification of the migration stimulating factor produced by fetal and breast cancer patient fibroblasts. Proc Natl Acad Sci USA 1989 ; 86 : 2438-42.

17. Liotta LA, Mandler R, Murano G, et al. Tumor cell autocrine motility factor. Proc Natl Acad Sci USA 1986 ; 83 : 3302-6.

18. Guirgis R, Margulies I, Taraboletti G Schiffmann E, Liotta L. Cytokine-induced pseudopodial protrusion is coupled to tumor cell migration. Nature 1987; 329 : 261-3.

19. Nabi IR, Watanabe H, Raz A. Identification of B16-F1 melanoma autocrine motility-like factor receptor. Cancer Res 1990 ; 50 : 409-14

20. Tamm I, Cardinale I, Krueger J, Murphy JS, May LT, Sehgal PB. Interleukin 6 decreases cell-cell association and increases motility of ductal breast carcinoma cells. J Exp Med 1989; 170 : 1649-69.

21. Nicolson GL. Organ specificity of tumor metastasis : role of preferential adhesion, invasion and growth of malignant cells at specific secondary sites. Cancer Metastasis Rev 1988 ; 7 : 143-88.

22. Edelman GM, Cunningham BA, Thiéry JP. Morphoregulatory molecules. New York : 1990. John Wiley, 1990.

23. Behrens J, Mareel MM, Van Roy FM, Birchmeier W. Dissecting tumor cell invasion : epithelial cells acquire invasive properties after the loss of uvomorulin-mediated cell-cell adhesion. J Cell Biol $1989 ; 108$ : 2435-47. cade d'événements. Des efforts ont été faits par de nombreuses équipes afin de caractériser un ou plusieurs gènes qui seraient responsables de l'acquisition de ces propriétés, mais ils n'ont pas été couronnés de succès [8].

Un certain nombre de gènes ou oncogènes (pour revue, voir [9]) ont la capacité de transformer des cellules. Ceux de la famille ras ont été le plus souvent caractérisés dans certaines tumeurs humaines [10]. Cependant toutes ces tumeurs ne sont pas métastatiques. Le rôle potentiel des oncogènes dans la cascade métastatique a été étudié dans de nombreux laboratoires en utilisant des modèles expérimentaux : des cellules transfectées avec un oncogène activé transformant (le plus souvent de la famille ras) ont été injectées à des souris nude. Dans la plupart des cas, outre la tumeur primaire, des métastases spontanées pulmonaires, hépatiques ou ganglionnaires ont été obtenues. Cependant, de nombreux résultats contradictoires ont aussi été publiés [11], démontrant que des cellules tumorigènes exprimant fortement un oncogène peuvent ne pas être métastatiques dans ce modèle. Il ressort de ces études que les capacités métastatiques des cellules dépendent grandement du type de cellule transformée, du type de l'oncogène transfecté (nature de la mutation activant le gène, par exemple), de l'environnement au sens large et enfin du microenvironnement cellulaire rencontré.

Les résultats cliniques qui permettraient de faire un lien entre oncogènes et métastase sont encore moins concluants et parfois même déconcertants ; c'est ainsi qu'il a été démontré que l'expression des oncogènes ras et $m y c$ pouvait être augmentée dans des tumeurs à des stades précoces de la progression (tumeurs bien différenciées par exemple) et complètement éteinte dans des tumeurs prolifératives ou métastatiques, alors que, dans la plupart des cas étudiés, l'oncogène activé est trouvé fortement exprimé dans la tumeur primaire et dans les métastases. La découverte de gènes suppresseurs des tumeurs laisse entrevoir la possibilité de définir des gènes suppresseurs de la métastase [12]. Une telle approche a été choisie pour identifier des ARN dont l'expression diminue au cours de l'acquisition du phénotype métastatique $[13,14]$ : le taux d'une protéine de $17 \mathrm{kDa}$, baptisée NM23, est ainsi considérablement réduit dans les tumeurs métastatiques alors que cette protéine est abondamment exprimée dans tous les tissus normaux et tumoraux non métastatiques.

- Facteurs de dissociation et de mobilité. Plusieurs équipes ont identifié des facteurs induisant une mobilité accrue de certaines lignées cellulaires normales et tumorales. Une classe de facteurs agit surtout sur des cellules normales ; l'un d'entre eux, appelé scatter factor (facteur de dispersion), serait composé de deux chaînes polypeptidiques de 30 et $57 \mathrm{kDa}$. Ce facteur qui induit la dissociation des épithéliums provoque également le déplacement de cellules isolées [15]. Un autre facteur sécrété par des fibroblastes fœtaux ou des fibroblastes de stroma tumoral a été partiellement purifié. Ce facteur, agissant par un mécanisme autocrine, augmente la mobilité des cellules introduites dans des gels de collagène [16].

Un autre facteur de mobilité, agissant également par un mécanisme autocrine, a été isolé à partir de surnageants de culture de mélanomes ; il correspond à un polypeptide de $55 \mathrm{kDa}$ [17]. Ce facteur, ainsi que d'autres facteurs qui lui sont apparentés, est actif sur de nombreux types de cellules tumorales et augmente leur mobilité in vitro. La liaison de ces facteurs avec des récepteurs membranaires spécifiques a pour effet d'activer des protéines $G$ intracellulaires sensibles à l'addition de toxine de $B$. Pertussis [18]. Très récemment, une glycoprotéine membranaire de $78 \mathrm{kDa}$ exprimée à la surface des mélanomes B16-F1 a été identifiée comme étant le récepteur liant le facteur autocrine de mobilité. Cette glycoprotéine est localisée essentiellement sur les lamellipodes des cellules en mouvement. Des anticorps monoclonaux dirigés contre la glycoprotéine $78 \mathrm{kDa}$ sont capables de mimer la liaison du facteur avec son récepteur et sont ainsi aptes à stimuler la mobilité des cellules de mélanome [19].

Par ailleurs, on a découvert récemment que l'interleukine- 6 pouvait 
induire l'arrêt de la prolifération, la dissociation et la mobilité cellulaire de lignées établies de carcinomes mammaires [20]. Ce résultat confirme ceux qui seront détaillés ici et montre que, dans certaines conditions, des facteurs de croissance peuvent jouer un rôle dans la mobilité des cellules tumorales.

Outre ces facteurs de mobilité tumorale, agissant souvent par un mécanisme autocrine, il semble qu'il existe des facteurs chimiotactiques ayant pour rôle d'attirer spécifiquement les cellules en cours de métastase vers les sites secondaires de progression tumorale [21]. Ces facteurs chimiotactiques, sécrétés par les organes cibles de la métastase, sont connus pour avoir des effets chimiocinétiques associés, favorisant ainsi la mobilité et le pouvoir invasif des cellules tumorales en cours d'extravasation. Cependant, aucun de ces facteurs n'a encore été caractérisé de façon satisfaisante.

- L'adhérence cellulaire. L'adhérence cellulaire comporte deux aspects différents (adhérence au substrat et adhérence intercellulaire [22]), qui tous deux interviennent sans doute dans le développement des métastases. Les cellules interagissent entre elles soit au moyen de molécules d'adhérence intercellulaire (ou CAM : cell adhesion molecules) soit, et ceci est particulièrement apparent dans les épithéliums et les tumeurs carcinomateuses qui en dérivent, au moyen de jonctions intercellulaires très différenciées (desmosomes, jonctions serrées, jonctions adhérentes, par.exemple). Si un certain nombre d'études qualitatives ou quantitatives ont échoué à montrer une modification du contenu en CAM et jonctions intercellulaires lorsque des tumeurs primaires étaient comparées avec leurs métastases, en revanche des études in vitro ont révélé que l'absence d'expression de certaines CAM à la surface des cellules cancéreuses était corrélée à un grand pouvoir invasif de ces cellules [23]. La contradiction entre ces résultats n'est peut-être qu'apparente et elle pourrait refléter le fait qu'une modulation transitoire de l'expression de ces molécules serait suffisante pour déclencher la dissociation cellulaire, ce qui entraînerait le départ des cellules dissociées hors de la masse tumorale. Ces cellules dissociées auraient alors une capacité $\mathrm{m} / \mathrm{s} n^{\circ} 5$ vol. 6 , mai 90 d'envahissement local et de métastase plus importante que les cellules emprisonnées dans la tumeur.

Par ailleurs, lors de leur transport lymphatique ou sanguin, les cellules tumorales ont tendance à former des agrégats (figure 2) : une des molécules adhésives impliquées dans cette agrégation serait une lectine endogène reconnaissant le galactose, cette lectine étant plus abondante à la surface des cellules ayant un pouvoir métastatique accru [24, 25].

D'autre part, l'adhérence intercellulaire spécifique pourrait intervenir également lors de l'arrêt des cellules tumorales dans les organes cibles de la métastase. Paget notait que les cellules tumorales ne peuvent se fixer et proliférer que dans certaines localisations précises, selon la loi du seed and soil. Une des premières étapes de ce processus de colonisation secondaire serait donc la reconnaissance spécifique entre des cellules tumorales migrant dans les vaisseaux sanguins ou lymphatiques et des molécules d'adhérence cellulaire exprimées sur les cellules endothéliales de certains organes. Grâce à cette interaction cellulaire, les cellules tumorales auraient la possibilité de se fixer dans ces organes privilégiés pour y proliférer ensuite [21, 26].

Un autre aspect de l'adhérence cellulaire est représenté par les interactions des cellules avec le substrat, assurées par la reconnaissance spécifique entre certains composants de la matrice extracellulaire et leurs récepteurs présents à la surface des cellules. Ces interactions interviendraient à plusieurs niveaux dans le processus métastatique, mais notons ici le rôle privilégié de certains substrats dans la migration des cellules tumorales : la fibronectine, composant interstitiel important, possède dans sa séquence protéique des sites polypeptidiques essentiels à la migration des cellules de mélanome. Ainsi, l'injection de peptides (Arg-Gly-Asp-Ser), correspondant à une des séquences d'attachement des cellules de mélanome sur la fibronectine, est-elle capable de réduire prodigieusement le développement des métastases provenant de la dissémination des cellules de mélanome [27]. De même, on a pu démontrer le rôle de la laminine, autre élément de la matrice extracel- lulaire, dans la migration de nombreux types de cellules cancéreuses [28]. Mais les rapports entre cellules métastatiques et tissu interstitiel sont d'autant plus compliqués à analyser que les cellules tumorales, qui peuvent utiliser des protéines de ce tissu comme substrat de migration, sont également capables de sécréter des enzymes protéolytiques dégradant la matrice extracellulaire.

- Les protéases. De nombreuses enzymes protéolytiques sont synthétisées par les cellules tumorales. Parmi elles, l'activateur du plasminogène, des hydrolases lysosomales et différentes collagénases [29], capables de dégrader divers composants de la matrice extracellulaire, pourraient contribuer au processus invasif. Ainsi les carcinomes in situ sont-ils entourés d'une lame basale riche en laminine et collagène de type IV qui peut être dégradée localement sous l'action de protéases tumorales comme la collagénase de type IV. Ce processus de dégradation pourrait expliquer les corrélations trouvées entre le taux de production de collagénase IV et le degré d'envahissement des cellules tumorales [30, 31]. De la même façon, l'activateur du plasminogène, qui transforme le plasminogène en plasmine, est capable d'activer des procollagénases et de dégrader la matrice extracellulaire. In vivo, la plasmine est détectée dans les zones d'infiltration des cancers du sein et du côlon [32, 33]. L'injection d'anticorps anti-activateur du plasminogène diminue le nombre de métastases obtenues après injection de cellules de carcinome [34] ; de même, la transfection d'ADNc codant pour des ARN messagers anti-sens capables d'inhiber spécifiquement les ARN cellulaires codant pour un inhibiteur naturel des collagénases augmente-telle la malignité des cellules transfectées [35]. Ces expériences suggèrent donc que la production de protéases joue un rôle essentiel dans les phases invasives du développement tumoral.

\section{Un modèle d'expérimentation in vitro}

D'après ce qui précède, il apparaît clairement que la difficulté à étudier le phénomène métastatique réside en 


\section{RÉFÉRENCES}

24. Raz A, Lotan R. Endogenous galactoside-binding lectins : a new class of functional tumor cell surface molecules related to metastasis. Cancer Metastasis Rev 1987 ; $6: 433-52$.

25. Raz A, Pazerini G, Carmi P. Identification of the metastasis-associated, galactoside-binding lectin as a chimeric gene product with homology to an IgE-binding protein. Cancer Res 1989 ; 49 : 3489-93.

26. Stoolman LM. Adhesion molecules controlling lymphocyte migration. Cell 1989 ; 56 : 907-10.

27. Humphries MJ, Olden K, Yamada KM. A synthetic peptide from the cellbinding domain of fibronectin inhibits experimental metastasis of murine melanoma cells. Science $1986 ; 233$ : 467-70.

28. Iwamoto Y, Robey FA, Graf J, et al. YIGSR, a synthetic laminin pentapeptide, inhibits experimental metastasis formation. Science 1987; 238 : 1132-4.

29. Mignatti M, Robbins E, Rifkin DB. Tumor invasion through the human amniotic membrane : Requirement for a proteinase cascade. Cell 1986; 47 : 487-98.

30. Liotta LA, Rao CN, Wewer UM. Biochemical interactions of tumor cells with the basement membrane. Ann Rev Biochem 1986 ; 55 : 1037-57.

31. Liotta LA, Stetler-Stevenson W. Metalloproteinases and malignant conversion : does correlation imply causality ? J Natl Cancer Inst $1989 ; 8$ : 556-7.

32. Clavel C, Chavanel G, Birembaut P. Detection of the plasmin system in human mammary pathology using immunofluorescence. Cancer Res 1986 ; 46 : 5743-7.

33. Burtin P, Chavanel G, André J. The plasmin system in human colonic tumors : an immunofluorescence study. Int $\mathrm{J}$ Cancer $1985 ; 35: 307-14$.

34. Ossowski L, Reich E. Antibodies to plasminogen activator inhibit human tumor metastasis. Cell 1983 ; 35 : 611-9. particulier dans l'extrême complexité du processus, qui met en jeu des caractéristiques cellulaires dépendant de l'étape à laquelle se trouve la cellule tumorale. Malgré tout, une propriété qu'il semble indispensable que la cellule tumorale acquière est la possibilité de se déplacer, propriété dont la cellule aura besoin à diverses étapes de la dissémination métastatique, soit pour quitter la tumeur primaire, soit au moment de s'installer dans les sites secondaires de progression tumorale. Quelles sont alors les modifications cellulaires et moléculaires nécessaires au passage d'un état immobile, tel qu'il peut se rencontrer au sein d'une tumeur solide, à un état de migration active? D'autre part, quels sont les signaux inducteurs de cette acquisition de mobilité ? Pour répondre à ces questions, un modèle expérimental a été développé, dans lequel la transition entre les états immobile et mobile a été étudiée. La lignée de carcinome vésical de rat, baptisée NBT-II [36], cultivée in vitro dans des conditions standard, a une morphologie typiquement épithéliale, formant une monocouche de cellules polygonales immobiles. Lorsque ces cellules sont soumises à l'action d'un facteur de croissance connu sous le nom de FGF (fibroblast growth factor) acide, les cellules se dispersent, migrent activement et adoptent une morphologie que l'on peut qualifier de fibroblastique, puisque les cellules s'allongent, s'aplatissent et émettent des pseudopodes [37]. De profonds bouleversements cellulaires, concernant en particulier toute l'architecture (le cytosquelette) de la cellule accompagnent les modifications observées. Les jonc- tions intercellulaires, assurant la continuité du feuillet épithélial, s'internalisent durant cette conversion, ce qui conduit à la dissociation cellulaire [38]. Grâce à ce modèle expérimental, il est donc possible de montrer que les cellules carcinomateuses sont capables de se dissocier et de se déplacer sous l'action d'un facteur physiologique, qui peut être sécrété, soit par les cellules tumorales ellesmêmes, soit par le stroma péritumoral. De plus, les cellules NBT-II ne forment pas de structures épithéliales et migrent lorsqu'elles sont cultivées, soit sous forme de cellules isolées [39], soit sous forme d'agrégats, sur des fibres de collagène de type I : les modifications cellulaires sont alors comparables à celles observées après induction par le FGF acide. Là encore, la transition est très spécifique du substrat utilisé et ne se produit pas lorsque le collagène est remplacé par d'autres composants de la matrice extracellulaire, comme la fibronectine ou la laminine [40]. Il apparaît donc que des protéines présentes dans les lames basales entourant les carcinomes ou dans le tissu conjonctif péritumoral sont capables d'induire et d'assurer le déplacement des cellules tumorales. Il est intéressant de noter que l'action combinée du FGF acide et d'un support collagénique conduit à une mobilité et une invasivité très accrue de ce carcinome (Tucker et al., manuscrit en préparation); une telle situation pourrait se produire in vivo.

\section{Conclusion et perspectives}

Depuis une vingtaine d'années, des efforts considérables ont été accom-
Figure 4. Dissociation et acquisition de mobilité des cellules d'un carci- $\triangleright$ nome vésical en culture in vitro. En conditions standard de culture, les cellules forment un tapis épithélial (a). Sous l'action du FGF acide ou de collagène, les cellules adoptent une morphologie fibroblastique $(b, c)$. Des expériences d'immunofluorescence réalisées avec des anticorps dirigés contre des protéines spécifiques des desmosomes mettent en évidence la dissociation cellulaire. Les desmosomes sont détectés à la périphérie cellulaire dans des conditions où les cellules conservent une morphologie épithéliale (d). Dans les conditions permettant l'acquisition de la mobilité et du phénotype fibroblastique, les cellules sont dissociées et les desmosomes ne sont plus que rarement détectés $(e, f)$. 


\section{Milieu standard}
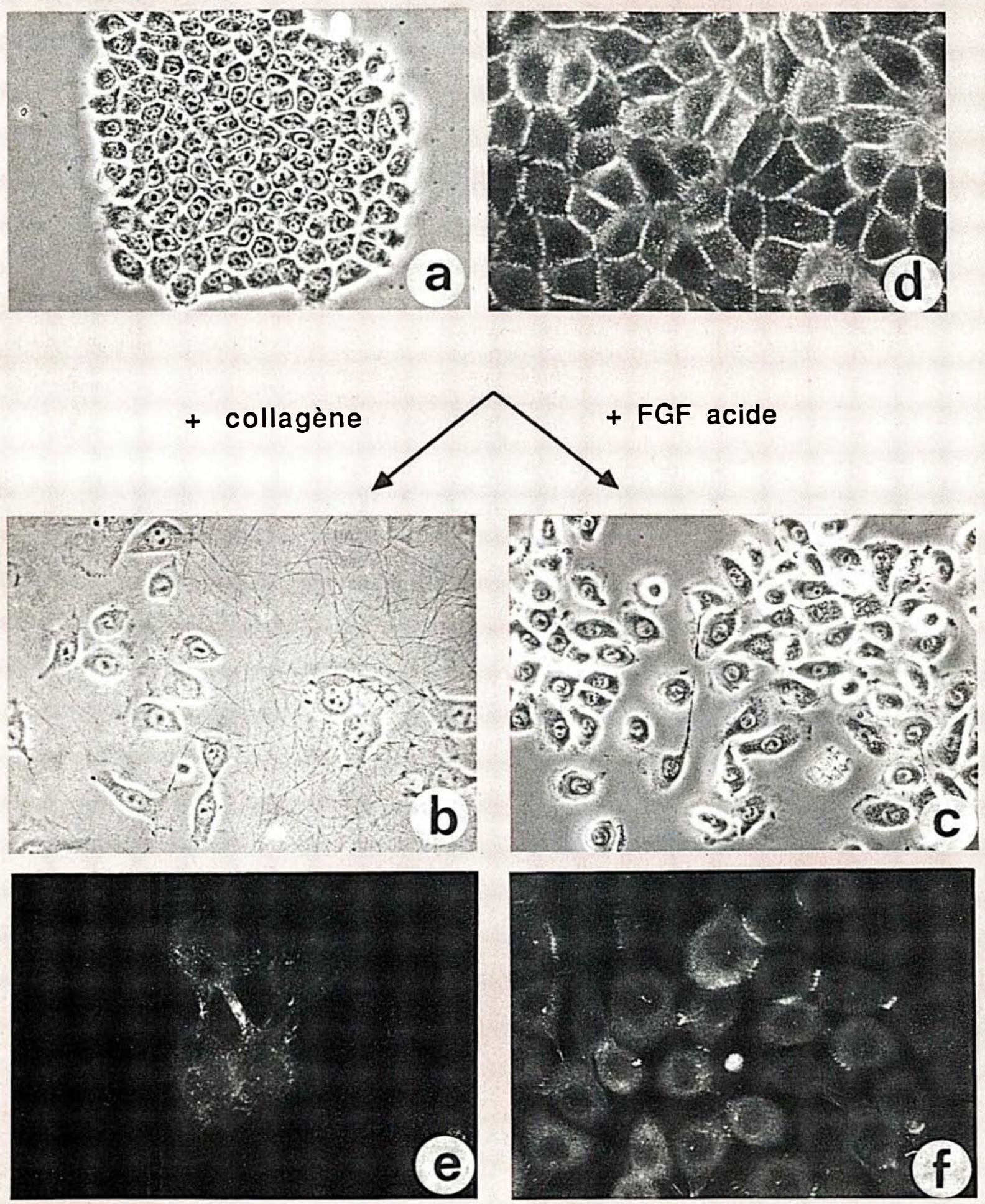


\section{RÉFÉRENCES}

35. Khokha R, Waterhouse P, Yagel S, et al. Antisense RNA-induced reduction in murine TIMP levels confers oncogenicity on Swiss 3T3. Science $1989 ; 243$ : 947-50.

36. Toyoshima K, Ito N, Hiasa Y, Kamamoto Y, Makiura S. Tissue culture of urinary bladder tumor induced in a rat by $\mathrm{N}$ butyl-N-(4-hydroxybutyl) nitrosamine : establishment of cell line, Nara Bladder Tumor II. J Natl Cancer Inst 1971 ; 47 : 979-85.

37. Vallés AM, Boyer B, Tucker GC, Badet J, Barritault D, Thiery JP. Acidic fibroblast growth factor is a modulator of epithelial plasticity. Proc Natl Acad Sci USA $1990 ; 87$ : 1124-8.

38. Boyer B, Tucker GC, Vallés AM, Franke WW, Thiery JP. Rearrangement of desmosomal and cytoskeletal proteins during the transition from epithelial to fibroblastoid organization in cultured rat bladder carcinoma cells. $J$ Cell Biol 1989; 109 : 1495-509.

39. Tchao R. Novel forms of epithelial cell motility on collagen and on glass surfaces. Cell Motility 1982 ; 4 : 333-41.

40. Tucker GC, Boyer B, Gavrilovic J, Emonard H, Thiery JP. Collagen-mediated dispersion of NBT-II rat bladder carcinoma cells. Cancer Res 1990 ; 50 : 129-35.

41. Thiery JP, Dufour S, Duband JL. Les fibronectines : une famille de glycoprotéines intervenant dans la morphogenèse et les migrations cellulaires. médecine/sciences 1987 ; $3: 316-25$

42. Carnemolla B, Balza E, Siri A, et al. A tumor-associated fibronectin isoform generated by alternative splicing of messenger RNA precursors. J. Cell Biol $1989 ; 108$ : 1139-48.

43. Spring J, Beck K, Chiquet-Ehrismann $\mathrm{R}$. Two contrary functions of tenascin : dissection of the active sites by recombinant tenascin fragments. Cell 1989 ; 59 : 325-34. plis pour comprendre la pathogénie des cancers. Dans le même temps, on a essayé d'analyser un des aspects de la tumorigenèse dont les conséquences cliniques sont les plus redoutables, à savoir le développement métastatique. Ces deux préoccupations scientifiques différentes pourraient finalement se rejoindre pour aboutir à une vision plus unifiée du développement tumoral. C'est ainsi que la découverte des oncogènes, et aujourd'hui des anti-oncogènes, pourra certainement avoir des retombées bénéfiques sur la compréhension du phénomène métastatique. Parallèlement à cette approche, certaines équipes ont cherché à utiliser des méthodes expérimentales ayant donné des résultats fructueux en cancérologie (par exemple, les protocoles qui ont été suivis pour mettre en évidence les oncogènes) pour trouver les gènes responsables du pouvoir métastatique. Il semble que cette tactique soit capable de donner des résultats. Par ailleurs, la recherche qui se fonde sur une analyse cellulaire et moléculaire fine de systèmes expérimentaux reproduisant une des étapes du processus métastatique est une autre voie de recherche possible. Cette approche pourra conduire à la notion que la répression ou l'activation de certains gènes cellulaires, par un mécanisme qu'il resterait alors à définir, est une phase essentielle dans l'acquisition du pouvoir métastatique.

Cet article n'a pas abordé le rôle de la réponse immunitaire dans le développement métastatique. Bien que les résultats dans ce domaine soient encore objets de controverses, il ne fait pas de doute que la thérapeutique immunologique reste un des grands espoirs dans le traitement des cancers et de leurs métastases

\section{Remerciements}

Les travaux du laboratoire de physiopathologie du développement bénéficient du soutien financier du Cnrs, de l'ENS, de l'ARC (6455) et de la Ligue nationale françaisc contre le cancer. Xavier Sastre a un contrat coopératif de l'institut Curie avec Jcan-Paul Thiery.

\section{Summary}

Metastasis of cancer cells

The metastastic cascade leads to the dissemination of cancer cells in secondary sites of tumor growth. Various cellular mechanisms triggering the acquisition of the metastatic potential have been studied using experimental systems that mimick the in vivo situation. It has been thus postulated that some modifications of cellular properties, such as the loss of intercellular adhesion, the activation of oncogenes, the induction of proteinase synthesis or secretion of motility factors may be involved in the pathogenesis of metastasis. Understanding the mechanisms by which tumor cells acquire a metastatic potential and discovering specific markers of this phenomenon might represent a promising tool to be used in the follow-up of cancer patients. 